\title{
High Frame Rate Ultrasonic Imaging through Fourier Transform using an Arbitrary Known Transmission Field
}

\author{
Hu Peng \\ University of Science and Technology of China
}

China

\section{Introduction}

Based on the study of limited array diffraction beams, a High Frame Rate (HFR) imaging method which uses a broadband pulsed plane wave, or array beams transmission field from a linear transducer array to illuminate the area to be imaged has been developed by Jian-yu LU. Echoes from the objects are received with the same transducer as is used in transmission. For each array beam parameter in a certain range, the received signals are weighted with that array beam and are summed up. The summations are Fourier transformed from time domain to frequency domain, and then processed further with the so called "parameter match" to produce the spectrum of the imaging. 2D and 3D images are constructed with inverse Fourier transformer respectively. In this way, the frame per transmission imaging rate is achieved.

Despite its advantages of high frame rate and high signal to noise ratio, the original HFR method has several drawbacks. It can only use the plane wave or the array beam transmission field, and is difficult to be ported for a non-array beam field, such as a cylindrical or spherical wave. Moreover, since the plane wave transmission field illuminates only a narrow area of its own width, the imaged area is quite small, and the only way to widen it up is to steer the transmission beams several times from different angles, which lowers the frame rate. Besides, the array beam field demands a linear transducer and a very complex weighting process.

Therefore, the HFR method needs to allow diffraction wave transmission fields in order to be practically useful. For example, it may use a cylindrical or spherical field and output sector format images like the conventional sector B mode ones, which have contributed a lot in diagnosing myocardial diseases.

In this chapter, an extended HFR method for 2D imaging is proposed. It allows all kinds of transmission field, including the cylindrical one and the spherical one, as well as the plane wave one. It is more general than the original HFR method.

The extended HFR method works mostly like the original one, except that 1 , it implements the weight-and-sum process through the Fourier transform; and 2, it iterates for each frequency in a certain range to obtain firstly a coarse image component at that frequency and then the refined one with the information of the transmission field removed. After the iteration the image components are summed up and that is the final image. 
In ultrasonic imaging systems, the cylindrical transducer, circle or curve transducer and linear transducer are commonly used. The advantages and disadvantages among them are different. One characteristic of the cylindrical or circle transducer is they can illuminate a sector or pyramidal area of the object. Therefore, in the following section, we extend the HFR method by using a cylindrical wave to illuminate an object. Mathematical formulas are derived and computer simulations are performed to verify the method. The method allows to increase the illumination area by using a transducer of a small footprint, which is important for applications such as cardiac imaging where acoustic window sizes are limited.

This Chapter is organized as follows. Firstly, the HFR ultrasonic imaging system based on the angular spectrum principle is introduced. In the flowing section, this system is extended. The extended HFR method allows all kinds of transmission field. Finally, a high frame rate 2D and 3D imaging system with a curved or cylindrical array is proposed.

\section{High Frame Rate ultrasonic imaging system based on the angular spectrum principle}

A kind of high frame rate (HFR) 2D and 3D imaging method was developed by Jianyu Lu in 1997. Because only one transmission is required to construct a frame of image, this method can reach an ultra high frame rate (about 3750 volumes or frames per second for biological soft tissues at a depth of $200 \mathrm{~mm}$ ). In this section, a new HFR method is presented in the view of angular spectrum. Compared with conventional dynamic focusing method which uses delay-and-sum processing and Lu's HFR method, which uses a kind of special weighting on the received signal, the new method only use the Fourier transform algorithm to construct image. So the system implementation of the method could be greatly simplified. During constructing image, several array beams with different parameters are used as transmitted signals, and the spectra of a frame of image is obtained by synthesizing the image spectrums related to different transmit event. The simulation result shows that the solution not only suppresses the sidelobe of system greatly and obtains the high quality image but also still keeps high frame rate to some extent.

\subsection{Theory}

The HFR method is based on one transmission event. In order to get the image of the object, the transducer transmits the limited diffraction beams to the object then the same transducer receives the echo signals reflected by the scatters and constructs image by Fourier transform. Fig.2.1 is diagram of the linear array used by HFR method. As the transducer emits the limited diffraction beams, the distribution of the field is

$$
p(x, y, z, k)=A(k) e^{i k z}
$$

where $p(x, y, z, k)$ means the acoustic pressure at the position $(x, y, z)$ under the certain wave numbered $k$, and $k=\omega / c=2 \pi f / c, f$ is frequency and $c$ is acoustic speed. $A(k)$ is the frequency spectrum of exciting signal.

If there are some scatters in the $z=z^{\prime}$ plane, the pressure for the scatters is

$$
\begin{aligned}
s\left(x, y, z_{i}, k\right) & =f\left(x, y, z_{i}\right) p\left(x, y, z_{i}, k\right) \\
& =f\left(x, y, z_{i}\right) A(k) e^{j k z_{i}}
\end{aligned}
$$


where $f\left(x, y, z_{i}\right)$ is the reflective coefficient function of the scatters. Using Fourier transform, we can get another expression for equation (2.2) in the angular spectrum domain:

$$
\begin{aligned}
& S\left(k_{x}, k_{y}, k, z_{i}\right)=\int_{x, y} s\left(x, y, z_{i}, k\right) e^{i k_{x} x+i k_{y} y} d x d y \\
& =\int_{x, y} f\left(x, y, z_{i}\right) A(k) e^{i k z_{i}} e^{i k_{x} x+i k_{y} y} d x d y
\end{aligned}
$$

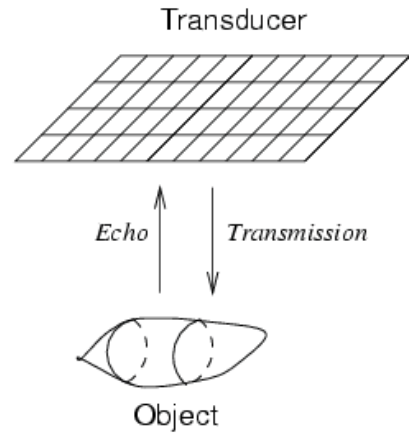

Fig. 2.1. Linear transducer array used in HFR

Because of the reflection of scatters, the echo signal represented by equation (2.2) or equation (2.3) propagates to the surface of the transducer. Based on the angular spectrum, it is easy to get the signal received by the transducer located in the plane $z=0$ in angular spectrum:

$$
R\left(k_{x}, k_{y}, k\right)=T(k) S\left(k_{x}, k_{y}, k, z_{i}\right) e^{i \sqrt{k^{2}-k_{x}^{2}-k_{y}^{2}}}
$$

Where $T(k)$ is the frequency response of the transducer. For simplicity, we assume

$$
k_{z}=\sqrt{k^{2}-k_{x}^{2}-k_{y}^{2}}
$$

From equation (2.4) and equation (2.3), the received signal can be represented further as follow

$$
R\left(k_{x}, k_{y}, k\right)=\int_{x, y} f\left(x, y, z_{i}\right) A(k) T(k) e^{i k_{z}^{\prime} z_{i}} e^{i k_{x} x+i k_{y} y} d x d y
$$

where

$$
k_{z}^{\prime}=k+\sqrt{k^{2}-k_{x}^{2}-k_{y}^{2}}
$$

The equation (2.6) means the signal, which is received by transducer located in the plane $z=0$, is come from echo signal produced by the scatters at the plane $z=z^{\prime}$. In fact the received signal comes from a lot of planes in the acoustic field. So it should be the summation of $R\left(k_{x}, k_{y}, k\right)$ over different depth, namely 


$$
\begin{aligned}
& R^{\prime}\left(k_{x}, k_{y}, k\right)=\int_{z} R\left(k_{x}, k_{y}, k_{z}\right) d z= \\
& \iiint_{x, y, z} f\left(x, y, z_{i}\right) A(k) T(k) e^{i k_{x} x+i k_{y} y+i k_{z}^{\prime} z_{i}} d x d y d z
\end{aligned}
$$

Assume

$$
F_{B L}\left(k_{x}, k_{y}, k_{z}^{\prime}\right)=R^{\prime}\left(k_{x}, k_{y}, k\right)
$$

where the subscript "BL" means "band-limited". From the spectrum $F_{B L}\left(k_{x}, k_{y}, k_{z}^{\prime}\right)$, the useful information of the object can be obtained by the inverse Fourier transform:

$$
f_{B L}(x, y, z)=F^{-1}\left(F_{B L}\left(k_{x}, k_{y}, k_{z}^{\prime}\right)\right)
$$

$F^{-1}(\cdot)$ is a inverse Fourier transform. From equation (2.10), the relationship between image $f_{B L}(x, y, z)$ and the object $f(x, y, z)$ is expressed as

$$
\begin{gathered}
f_{B L}(x, y, z)=\int_{x, y, x} d x d y d z f(x, y, z) \int_{k_{x}, k_{y}, k_{z}} A(k) T(k) e^{-i k_{x}\left(x^{\prime}-x\right)-i k_{y}\left(y^{\prime}-y\right)-i k_{z}\left(z^{\prime}-z\right)} d k_{x} d k_{y} d k_{z} \\
=\int_{x, y, z} d x d y d z f(x, y, z) p\left(x-x^{\prime}, y-y^{\prime}, z-z^{\prime}\right) \\
=f(x, y, z) \otimes p(x, y, z)
\end{gathered}
$$

Where the function $p(x, y, z)$ is defined as

$$
\begin{aligned}
& p(x, y, z)=F^{-1}\left(P\left(k_{x}, k_{y}, k_{z}^{\prime}\right)\right) \quad \text { and } \\
& P\left(k_{x}, k_{y}, k_{z}^{\prime}\right)=[A(k) T(k)]_{k_{x}, k_{y}, k_{z}^{\prime}}
\end{aligned}
$$

From the equation (2.11) and (2.12), we can see that if the size of the aperture is infinite, the image is the result of the convolution between object reflection coefficient and the function $p(x, y, z)$. So $p(x, y, z)$ is the point spread function (PSF) of the imaging system, which is determined by the excited signal and the frequency response of transducer. Obviously under the condition that $k$ is infinite and $A(k) T(k)$ is equal to one, $p(x, y, z)$ turns to be Dirac delta function and $f_{B L}(x, y, z)$ is the object $f(x, y, z)$. Generally, The bandwidth of $T(k)$ and $A(k)$ is limited and $p(x, y, z)$ is pulse in three dimension. So $f(x, y, z)$ only presents some useful information of the object.

\subsection{Simulation results}

In equation (2.8), function $R^{\prime}\left(k_{x}, k_{y}, k\right)$ is the received signal by transducer in the domain of frequency spectrum $k$ and the domain of space spectrum $\left(k_{x}, k_{y}\right)$. In practice, the received signal is expressed by $r^{\prime}\left(x_{e}, y_{e}, t\right)$ in the domain of time $t$ and the domain of space $(x, y)$. So in the first step the algorithm 3D Fourier transform is used in order to change $r^{\prime}\left(x_{e}, y_{e}, t\right)$ to $R^{\prime}\left(k_{x}, k_{y}, k\right)$. It means the weighting process can be realized by Fourier transform over transducer surface and the time parameter.

But there is still a little difference between Fourier transform and the weighting process. First we know the number of wave $k$ is positive for constructing imaging, but the result of 
Fourier transform contains information of positive and negative $k$. Secondly the results of Fourier transform include the information part which corresponds to $\left|k_{x}\right|>k_{\max }$ and $\left|k_{y}\right|>k_{\max }$, and obviously the part has no physics meaning for the weighting result $R^{\prime}\left(k_{x}, k_{y}, k\right)$.

Considering the two condition, $R^{\prime}\left(k_{x}, k_{y}, k\right)$ can be obtained from the modified Fourier transform results of the received signal $r^{\prime}(x, y, t)$ under the condition below:

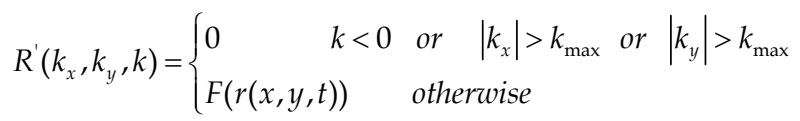

$F^{-1}(\cdot)$ is a inverse Fourier transform. Based on the study above, the system of HFR method can be simplified into Fig.2.2.

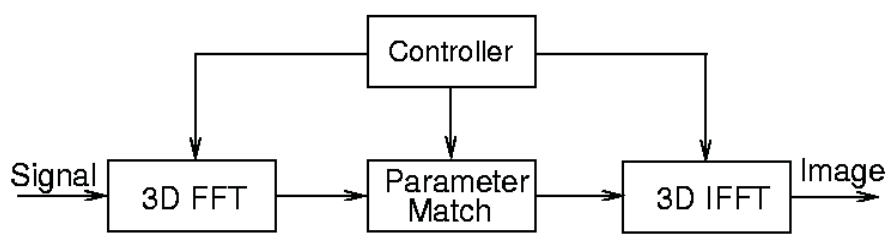

Fig. 2.2. The new solution to the realization of HFR method, which consists of three parts mainly, two 3D FFT and parameter match

In Fig.2.2 the system consists of three parts, two FFT chips and one parameter match chip. The received signal is imputed into the first FFT chip to get the signal $R^{\prime}\left(k_{x}, k_{y}, k\right)$, then processed by parameter match unit which changes $R^{\prime}\left(k_{x}, k_{y}, k\right)$ to the spectrum $F_{B L}\left(k_{x}, k_{y}, k_{z}^{\prime}\right)$ of the image, and at last step the image $f_{B L}(x, y, z)$ is obtained from $F_{B L}\left(k_{x}, k_{y}, k_{z}^{\prime}\right)$ by the second FFT chip.

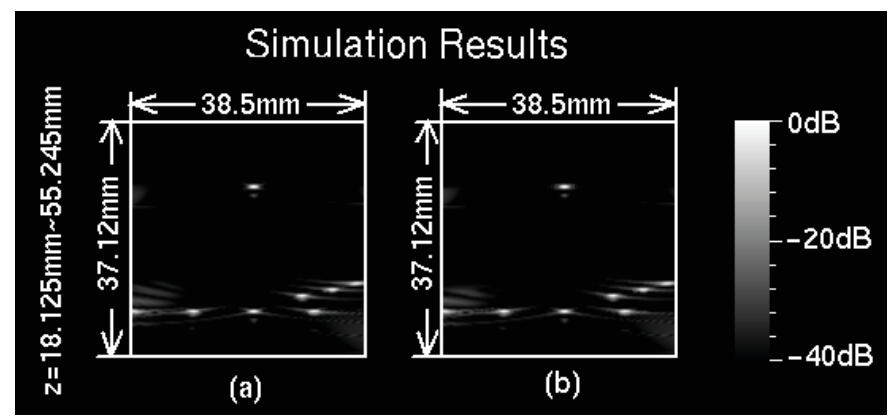

Fig. 2.3. Simulation of $2 \mathrm{D}$ B-mode image with log compressed over $40 \mathrm{db}$ scale. (a) is obtained by original HFR method, and (b) is obtained by the new method

To verify the new process, a simulation in two dimension was performed to construct image by the HFR method. In the simulation, the phantom consists of eight point scatters objects. The linear array transducers is a $2.5 \mathrm{MHz}$ array of 64 elements and a dimension of $38.4 \mathrm{~mm}$ with an inter-element space of $0.6 \mathrm{~mm}$. Two-way (pulse-echo) spectra of the arrays are assumed to be proportional to the Blackman window function with a fractional bandwidth 
of about $81 \%$ that is typical for a modern array. The simulation results shows in Fig. 2.3 and Fig.2.4. In the figures, Fig.2.3a is obtained by original HFR process (IEEE Trans on UFFC, 44(4), 1997, pp. 839-856) and Fig.2.3b is obtained by the new process. It can be seen that the two results are the same.

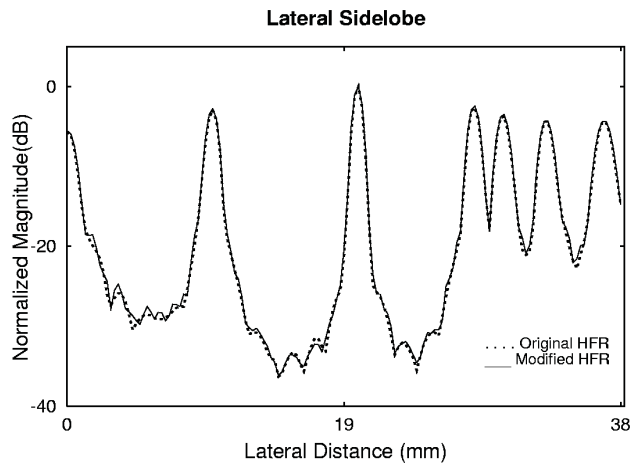

Fig. 2.4. The sidelobe along lateral direction

\subsection{Conclusion}

This section presents a theory analysis, which is based on angular spectrum principle, to simplify the HFR imaging system presented by Lu. Besides a new imaging mode is proposed, which use several transmission events to synthesize the image. In every transmission event, array beam with different parameters is used as excited signal. The constructed image has very high resolution and contrast, and meanwhile the imaging system still hold high frame rate to some extent.

\section{Construction of High Frame Rate ultrasonic images with Fourier transform in any kind of acoustic field}

In HFR method, a plane wave was used to illuminate an area for either 2D or 3D imaging. The drawback of this method is that the area illuminated is only as wide as the size of the aperture of the array transducer. In this section, a generic HFR method is developed. 2D high frame rate images can be constructed using the Fourier transform with a single transmission of an ultrasound pulse from an array under different transmission filed as long as the transmission filed is known. To verify our theory, computer simulations have been performed in the non-plane wave field. The field is cylindrical field defined by zero order Hankle function and produced by a linear array. The image with sector format and lower sidelobes is obtained. The simulation results are consistent with our theory.

\subsection{Theories}

For simplicity, we discuss our new method in the two dimension (2D). Let us assume that there is a linear array at the position $z=0$ (Fig.3.1), and the transmitted field is $p(x, z, k)$ where $k=2 \pi f / c . f$ means frequency and $c$ is acoustic speed. If there is a scatter at the position which the coordination is $(x, z)$, and the reflection coefficient is $f(x, z)$, The echo signal, which object scatter reflects, is as follow: 


$$
f^{\prime}(x, z, k)=f(x, z) p(x, z, k) T(k)
$$

where $T(k)$ is spectra, which is related to the spectrum of excited signal and the frequency response of the transducer.

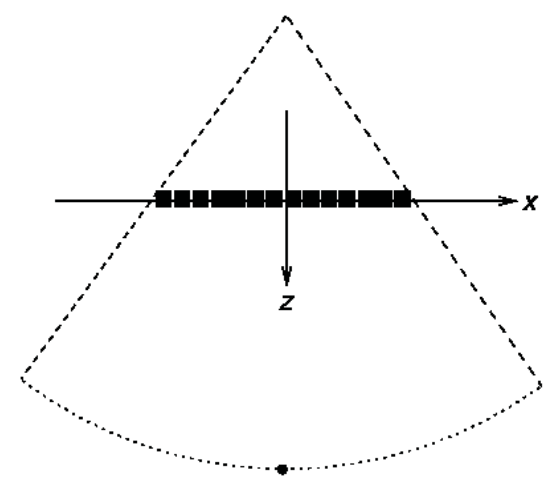

Fig. 3.1. The diagram of the transducer

The received signal to an element of the transducer can be obtained by the equation (3.2)

$$
r\left(x_{e}, z_{e}=0, k\right)=\int_{x, z} f^{\prime}(x, z, k) H\left(x, z ; x_{e}, z_{e}=0, k\right) d x d z
$$

Here $\left(x_{e}, z_{e}=0\right)$ is the position's coordination of an element of the transducer, $k$ is wave number, $H\left(x, z ; x_{e}, z_{e}=0, k\right)$ is transmission function, which is determined by RayleighSommerfield diffraction theory and presents the relationship between source point $(x, y)$ and observed point $\left(x_{e}, y_{e}\right)$. The function $r\left(x_{e}, z_{e}=0, k\right)$ means the received signal echoed by the object to be imaged.

Under a certain frequency component $k$, Using signal $e^{j k_{x} x_{e}}$ to weight the received signal

$$
\begin{aligned}
& R\left(k_{x}, k_{z}\right)=\int_{l} r\left(x_{e}, z_{e}=0, k\right) e^{j k_{x} x_{e}} d x_{e} \\
& =\int_{l} d x d z f^{\prime}(x, z, k) \int_{l} e^{j k_{x} x_{e}} H\left(x, z ; x_{e}, z_{e}=0, k\right) d x_{e}
\end{aligned}
$$

If the $l$, the size of the transducer is infinite, the result of the integrate over $l$ is

$$
\int e^{j k_{x} x_{e}} H\left(x, z ; x_{e}, z_{e}=0, k\right) d x_{e}=e^{j k_{x} x+j k_{y} y}
$$

Here

$$
k_{z}^{2}=\sqrt{k^{2}-k_{x}^{2}}
$$

This means that the transducer at the position $z=0$, which is excited by the weight signal $e^{j k_{x} x_{e}}$, produces the plane wave $e^{j k_{x} x+k_{y} y}$ in the field. From the equation (3.4) and (3.4), we have 


$$
R\left(k_{x}, k_{z}\right)=\int_{x, z} f^{\prime}(x, z, k) e^{i k_{x} x+i k_{z} z} d x d z
$$

Make inverse Fourier transform for the weighted signal $R\left(k_{x}, k_{z}\right)$ to get the imaging under the frequency component $k$ and the transmitted filed $p(x, z, k)$;

$$
f^{\prime}\left(x^{\prime}, z^{\prime}, k\right)=F\left\{R\left(k_{x}, k_{z}\right)\right\}
$$

where $F\{\cdot\}$ is Fourier transform. Remove the information of transmitted filed.

$$
f^{\prime \prime}\left(x^{\prime}, z^{\prime}, k\right)=f^{\prime}\left(x^{\prime}, z^{\prime}, k\right) p^{-1}\left(x^{\prime}, z^{\prime}, k\right)
$$

Sum the imaging $f^{\prime \prime}\left(x^{\prime}, z^{\prime}, k\right)$ over all frequency components to get final imaging.

$$
f^{\prime \prime \prime}\left(x^{\prime}, z^{\prime}\right)=\sum_{k} f^{\prime \prime}\left(x^{\prime}, z^{\prime}, k\right)
$$

We can prove that equation (3.9) is a good approximation of the object function $f(x, z)$. Especially when the transmitted filed is plane wave, it is the original HFR method.

\subsection{Simulation results}

From the theoretical analysis above, the simulation process is divided into several steps as follows:

1. According to the transmitted signal and the boundary of the transducer, calculate the distribution of the acoustic filed $p(x, z, k)$;

2. According to the equation (3.3) and (3.5), using signal $e^{j k_{x} x_{e}}$ to weight the received signal $r\left(x_{e}, z_{e}=0, k\right)$ to get the spectrum signal $R\left(k_{x}, k_{z}\right)$;

3. Using equation (3.7) to get imaging $f^{\prime}(x, z, k)$, which is at the frequency component $k$;

4. Remove the imaging's phase caused by $p(x, z, k)$ according to equation (3.8) to get signal $f^{\prime \prime}(x, z, k)$;

Sum the imaging $f^{\prime \prime}(x, z, k)$ for all frequency components to get final imaging $f^{\prime \prime \prime}(x, y)$ by equation (3.9);

Fig.3.1 shows the block diagram of the experiment. Assume the transmitted field is cylindrical function determined by zero order Hankel function. By adjusting the phase and amplitude of excited signal over the linear transducer according to the equation (3.10), the linear transducer produces the transmission filed as follows:

$$
p(x, z, k)=\frac{e^{j k \sqrt{x^{2}+z^{2}}}}{\sqrt{k} \sqrt{x^{2}+z^{2}}}
$$

The number of the transducer arrays is 128 . The length of the transducer is $37 \mathrm{~mm}$. The central frequency of the transducer is $2.5 \mathrm{MHz}$ and the bandwidth is about 80 percent of the central frequency. The frequency response function $T(k)$ is assumed to be Blackman window, which is adopted in most literature's simulation condition. The image is produced at the depth which $z$ is equal to $50 \mathrm{~mm}$. Fig. 3.2 is the result of the simulation for one scatters located at $(0,50)$. Fig3.2.a is the images of the object, which is Log compressed over $40 \mathrm{db}$. In 
order to observe the sidelobes, Fig3.2.b gives the plot line along lateral direction. Fig.3.2c gives the plot line along axial direction. From the figures, we can see that the sidelobes are about below $-40 \mathrm{db}$, which the resolution is about $1.2 \mathrm{~mm}$ in the lateral direction, and 0.8 in the axial direction. Fig.3.3 is another result of the simulation for seven scatters. The scatters are on the part of a circle, among which the central point scatters is equal to $50 \mathrm{~mm}$ far away from the surface of transducer. Fig3.3.a is the imaging of the object, which is Log compressed over 40db. Fig3.3.b shows the sidelobes along $x$ direction. Though the size of array is only $37 \mathrm{~mm}$, the distance in the images along lateral direction from left point scatter to the right scatter is about $58 \mathrm{~mm}$, which is larger than the size of the transducer. Obviously the imaging is impossible to be obtained for original HFR method.

a
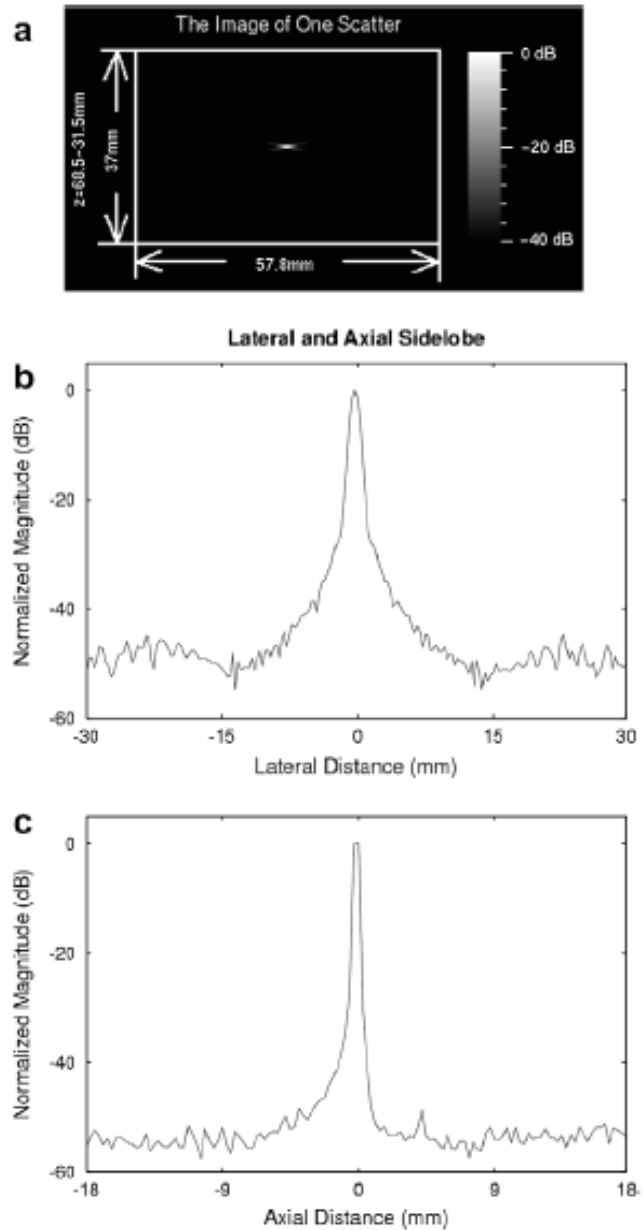

Fig. 3.2. Simulation results of Fig1. 1's one scatterer. (a). The $40 \mathrm{db}$ log compressed image and (b) and (c) sidelobes along the lateral and the axial direction 
a

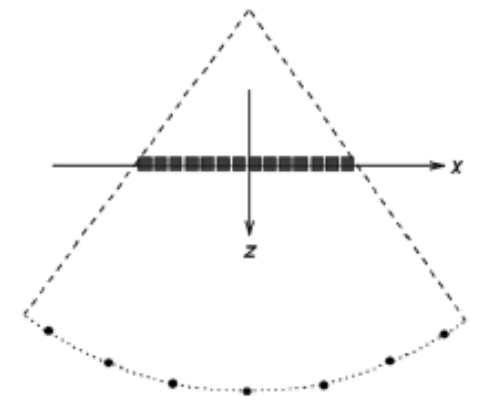

b
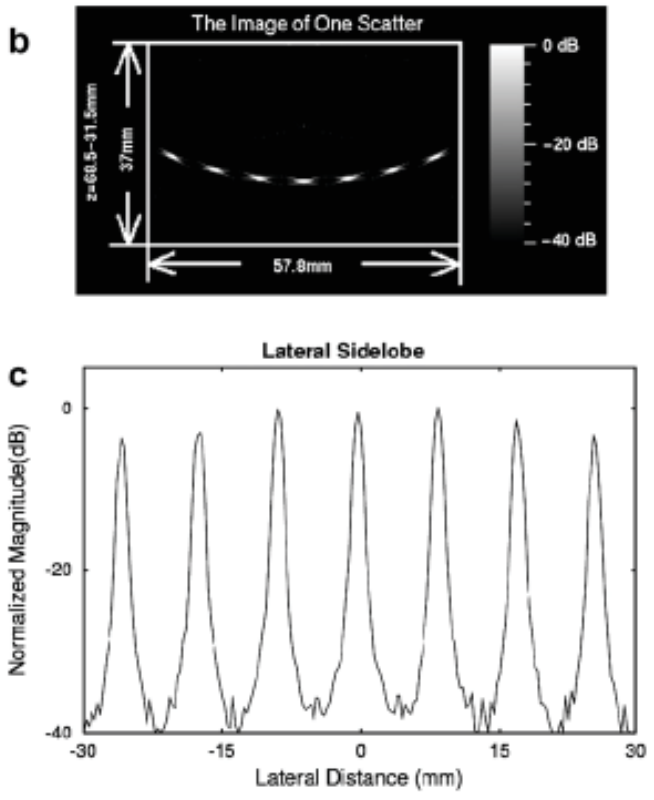

Fig. 3.3. Simulation results of seven scatters. (a). The diagram of the transducer and seven scatterers, (b). the $40 \mathrm{db} \log$ compressed image, (c). the sidelobes along $\mathrm{x}$ direction

\subsection{Conclusion}

Though the method is analyzed in the two dimensions, it can be obviously used in the three dimensions. So the method gives an effective way to construct images with sector form (2D) and pyramidal form (3D) by the linear array based on the Fourier transformer. Like the original HFR method, the system can construct images with only one time transmission, and the quality of the imaging is high. Compared to the original Fourier transform method, it is effective in any kind of acoustic field, though the principle of the method and the original HFR method is the same. In original HFR method, the kernel function of the Fourier transform contains the information of the transmission field because the transmission waves and weighting waves are the same kind beams, which belong to array beams. In our new method we extend the kind of transmission field from plane wave or array beams to other 
kind wave, such as cylindrical wave. As the weighting signal is not the same form as the transmission filed, it is difficult to combine the transmission filed and weighted signal together in the kernel function of the Fourier transform. As a result we have to repeat the Fourier transform process under different frequency component and make the summation over different frequency results. So the shortcoming of the method is obvious compared to the original HFR method, namely its quantity of the computation is high. If some kind of quick arithmetic is found, the method will be more effective in practice.

Because the original HFR method assumes the transmission filed to be the plane wave or array beams although it is impossible actually due to diffraction property in physics, the assumption makes results obtained by original HFR method a little disturbed when the distance between object and transducer is some large. For our method if the transmission filed is pre-known exactly by some method, such as simulation or measurement, the better results can be obtained because the new method can cancel the effects of transmission filed.

\section{High Frame Rate 2D and 3D imaging with a curved or cylindrical array}

The cylindrical transducer, circle or curve transducer and linear transducer are commonly used in ultrasonic imaging system. The advantages and disadvantages between them are different. One characteristic of the cylindrical or circle transducer is the transducer can illuminate a sector or pyramidal area of the object. The scanning format is primarily useful for cardiac imaging to avoid interference from the ribs. Since this kind of transducer is nonlinear transducer, the method of constructed images is a little different from the linear transducer's method.

\subsection{Theoretical preliminaries}

In the section, a new imaging method (Fourier method and radial matched filter) for a pulse system will be developed and formulas for construction of 2D and 3D images will be derived with zero order Hankle function.

\subsubsection{D images construction}

To simplify the analysis, we assume that the sampling of the array along each direction is regarded as continuous, our results, based on this assumption, should closely approximate that of a sampled aperture as long as the Nyquist criterion is met to avoid spatial aliasing . A sufficient condition for this criterion to be satisfied is a half-wavelength spacing of elements along the arrays. For simplicity, we will also neglect the diffraction patterns of the individual elements; they are assumed to be behaving as point sources and receivers. Although we assume continuously sampled, the simulation results shows similar principles can be applied to arrays of discrete elements of finite size.

For the generality, we discuss the method in three-dimension in the cylindrical coordinate system. Fig.4.1 shows a cylindrical transducer. Though the filed produced by cylindrical transducer is much more complex than the plane wave, we still can get simple form under some reasonable assumption. The simplest mode of the filed form produced by the cylindrical transducer is zero order Hankle function. If the $k r$ is relatively large, the acoustic pressure, which is presented by zero order Hankle function, can be estimated by:

$$
p(r, k) \approx A(k) \frac{e^{i k r}}{\sqrt{k r}}
$$


Where $k$ is wave number, $r=\sqrt{x^{2}+y^{2}}$ represents radial coordinate, $A(k)$ is related to the spectrum of the signal and the response of the transducer frequency and can be presented by the Blackman windows .

Based on the Rayleigh-Sommerfeld diffraction theory, the received signal for an element for all scatterers is easily given by

$$
\begin{aligned}
& R\left(k, \theta_{e}, z_{e}\right)=\frac{1}{i \lambda} \times \\
& \iiint_{V} r d r d \theta d z f(r, \theta, z) p(r, k) T(k) \frac{e^{i k \sqrt{r^{2}+r_{e}^{2}-2 r r_{e} \cos \left(\theta_{e}-\theta\right)+\left(z_{e}-z\right)^{2}}}}{\sqrt{r^{2}+r_{e}^{2}-2 r r_{e} \cos \left(\theta_{e}-\theta\right)+\left(z_{e}-z\right)^{2}}} \cos \left(\vec{n}_{1}, \vec{n}_{2}\right)
\end{aligned}
$$

where $\lambda$ is wavelength, and $\lambda=2 \pi / k . \theta$ is azimuthal angle, $z$ is axial axis, which is perpendicular to the plane defined by $r$ and $\theta . \sqrt{r^{2}+r_{e}^{2}-2 r r_{e} \cos \left(\theta_{e}-\theta\right)+\left(z_{e}-z\right)^{2}}=\left|\vec{r}_{e}-\vec{r}\right|$ is the distance between the scatterer and the transducer element, where $\vec{r}_{e}=\left(r_{e}, \theta_{e}, z_{e}\right)$ is the coordinate of transducer element, $\vec{r}=(r, \theta, z)$ is the coordinate of the scatters. $f(r, \theta, z)$ is the function of reflection coefficient of the object, $R=\left(k, \theta_{e}, z_{e}\right)$ means the received signal for the element at $\vec{r}_{e}, \vec{n}_{1}$ is an unit vector which direction is from $\left(0, \theta_{e}, z_{e}\right)$ to $\left(r_{e}, \theta_{e}, z_{e}\right)$, and $\vec{n}_{2}$ is another unit vector which the direction is from $(r, \theta, z)$ to $\left(r_{e}, \theta_{e}, z_{e}\right)$. Our objective is to image the reflectivity function $f(r, \theta, z)$, which is the inverse problem of equation (4.2). After some mathematical manipulations, one can easily find the following

$$
\begin{aligned}
& R\left(k, \theta_{e}, z_{e}\right)=\frac{\sqrt{k}}{i 2 \pi} A(k) T(k) \times \\
& \iint_{V} d r d \theta d z f(r, \theta, z) \frac{e^{i k r+i k \sqrt{r^{2}+r_{e}^{2}-2 r r_{e} \cos \left(\theta_{e}-\theta\right)+\left(z_{e}-z\right)^{2}}}}{r^{2}+r_{e}^{2}-2 r r_{e} \cos \left(\theta_{e}-\theta\right)+\left(z_{e}-z\right)^{2}} \sqrt{r}\left(r \cos \left(\theta_{e}-\theta\right)-r_{e}\right)
\end{aligned}
$$

Even if Equation.(4.3) is similar to Equation.(4.2), it is still different because the equation (4.3) includes cylindrical field information, and based on which the image can be constructed by only one transmission. .... It is obvious that equation (4.3) is the convolution form over $\theta, z$, so we have

$$
R\left(k, \theta_{e}, z_{e}\right)=\int_{r_{e}}^{\infty} d r f(r, \theta, z) *_{\theta, z} h(k, r, \theta, z)
$$

where $*_{\theta, z}$ means convolution operator over $\theta, z \cdot h(k, r, \theta, z)$ is defined as

$$
h(k, r, \theta, z)=\frac{\sqrt{k}}{i 2 \pi} A(k) T(k) \frac{e^{i k r+i k \sqrt{r^{2}+r_{e}^{2}-2 r r_{e} \cos \theta+z^{2}}}}{r^{2}+r_{e}^{2}-2 r r_{e} \cos \theta+z^{2}} \sqrt{r}\left(r \cos \theta-r_{e}\right)
$$

The equation (4.5) is system transform function, which transforms the object function $f(r, \theta, z)$ to the received signal $R\left(k, \theta_{e}, z_{e}\right)$. In the study, the exact form is used to construct image instead of an approximate form.

From equation (4.4), using Fourier transform theory, we have another expression in spectrum $k_{\theta}, k_{z}$ domain. 


$$
\tilde{R}\left(k, k_{\theta}, k_{z}\right)=F_{\theta, z}\{R(k, \theta, z)\}=\int_{r_{e}}^{\infty} d r \tilde{F}\left(r, k_{\theta}, k_{z}\right) \tilde{H}\left(k, r, k_{\theta}, k_{z}\right)
$$

where $\tilde{R}\left(k, k_{\theta}, k_{z}\right)$ is the Fourier transform of $R(k, \theta, z)$ in terms of $\theta, z, \tilde{H}\left(k, r, k_{\theta}, k_{z}\right)$ is the Fourier transform of $h(k, r, \theta, z)$. It is clear that Eq. (4.6) establishes a relationship between the Fourier transform of measured signal and the Fourier transform of object function. However, this relationship is established through the integration over $r$, which is the radial axis of the object function in the cylindrical coordinates. In the following section, we will use some mathematical manipulation to find and establish a more direct relationship between the Fourier transforms of these two functions.

From (4.5), it is clear that the filter function $\tilde{H}\left(k, r, k_{\theta}, k_{z}\right)$ contains an oscillating term of $\mathrm{k}$ and $\mathrm{r}$. This term may play a role of decreasing the integration in terms of either $\mathrm{k}$ or $\mathrm{r}$. If such oscillation term can be removed under some conditions, we may be able to construct images. Multiplying the conjugate of $\tilde{H}\left(k, r, k_{\theta}, k_{z}\right), \tilde{H}^{*}\left(k, r^{\prime}, k_{\theta}, k_{z}\right)$, to both sides of (4.6), we have:

$$
\tilde{R}\left(k, k_{\theta}, k_{z}\right) \tilde{H}^{*}\left(k, r^{\prime}, k_{\theta}, k_{z}\right)=\int_{r_{e}}^{\infty} d r \tilde{F}\left(r, k_{\theta}, k_{z}\right) \tilde{H}\left(k, r, k_{\theta}, k_{z}\right) \tilde{H}^{*}\left(k, r^{\prime}, k_{\theta}, k_{z}\right)
$$

Integrating over wave number $k$ for on both side of (4.7), one obtains:

$$
\begin{gathered}
\tilde{R}^{\prime}\left(r^{\prime}, k_{\theta}, k_{z}\right)=\int_{-\infty}^{\infty} d k \tilde{R}\left(k, k_{\theta}, k_{z}\right) \tilde{H}^{*}\left(k, r^{\prime}, k_{\theta}, k_{z}\right) \\
=\int_{r_{e}}^{\infty} d r \tilde{F}\left(r, k_{\theta}, k_{z}\right) G\left(r, r^{\prime}, k_{\theta}, k_{z}\right)
\end{gathered}
$$

where

$$
G\left(r, r^{\prime}, k_{\theta}, k_{z}\right)=\int_{-\infty}^{\infty} d k \tilde{H}\left(k, r, k_{\theta}, k_{z}\right) \tilde{H}^{*}\left(k, r^{\prime}, k_{\theta}, k_{z}\right)
$$

Equation (4.8) establishes a relationship between the measured signal, $\tilde{R}^{\prime}\left(r^{\prime}, k_{\theta}, k_{z}\right)$, which is known, and the Fourier transform of the object function, $\tilde{F}\left(r, k_{\theta}, k_{z}\right)$. After inverse Fourier transform over $k_{\theta}, k_{z}$ for equation (4.8), we have

$$
f^{\prime}(r, \theta, z)=F_{k_{\theta}, k_{z}}^{-1}\left\{\tilde{R}^{\prime}\left(r, k_{\theta}, k_{z}\right)\right\}=\int_{r_{e}}^{\infty} d r f(r, \theta, z) *_{\theta, z} g\left(r, r^{\prime}, \theta, z\right)
$$

What is the relationship between $f^{\prime}(r, \theta, z)$ and $f(r, \theta, z)$ ? In order to answer the question, let us consider the function $g\left(r, r^{\prime}, \theta, z\right)$, which is inverse Fourier transform of $G\left(r, r^{\prime}, k_{\theta}, k_{z}\right)$ in (4.9) and see its role in the constructed images. Fig4.2 and Fig4.3 shows the simulation of the distribution of the function $g\left(r, r^{\prime}, \theta, z\right)$. From the results of the simulation, two important properties can be seen. First it is clear that $g\left(r, r^{\prime}, \theta, z\right)$ peaks sharply only when $r$ is equal to $r^{\prime}$. Second, $g\left(r, r^{\prime}, \theta, z\right)$ is symmetric to both $r^{\prime}$ and $r$. This gives us the following approximate relationship:

$$
\left|g\left(r, r^{\prime}, \theta, z\right)\right| \approx\left|g^{\prime}\left(r-r^{\prime}, \theta, z\right)\right|
$$


From equation (4.10) and (4.11), we can reasonably assume:

$$
f^{\prime}(r, \theta, z)=\int_{r_{e}}^{\infty} d r f(r, \theta, z) *_{\theta, z} g^{\prime}\left(r-r^{\prime}, \theta, z\right)=f(r, \theta, z) *_{r, \theta, z} g^{\prime}(r, \theta, z)
$$

Obviously the function $g^{\prime}(r, \theta, z)$ can be treated as the point spread function of the imaging system. For a perfect imaging system, $g^{\prime}(r, \theta, z)$ is a Dirac-Delta function in space domain $(r, \theta, z)$. So the image $f^{\prime}(r, \theta, z)$ is object function $f^{\prime}(r, \theta, z)$. From the simulation in Figs.4.2 and 4.3 we see that $f^{\prime}(r, \theta, z)$ has a sharp point spread function in the space domain $(r, \theta, z)$ (please note that only one-way PSF is shown in Figs. 4.2 and 4.3 for high frame rate imaging), which is similar to the Derac-Delta function. Based on the analysis above, we have the answer for the question above:

$$
f^{\prime}(r, \theta, z) \approx f(r, \theta, z)
$$

\subsubsection{D image construction}

A 2D image in any orientation (including both B-mode and C-mode images) can be readily obtained from 3D images with equation (4.6), (4.8) and (4.10). However, 3D imaging is more complex and generally requires more computation. In the following, the formulas that are simplified from (4.6), (4.8) and (4.4.10) and are suitable for conventional B-mode imaging and a $\mathrm{C}$-mode imaging will be derived. In B-mode imaging, objects are assumed to be independent of $z$ (along the axial direction) and in C-mode imaging, objects are assumed to be a thin layer located at a radial direction $r=r_{0}$ away from the transducer, where $r_{0}$ is a constant.

C-mode imaging assumes the object function $f(r, \theta, z)$ represents a thin layer that is in parallel with the surface of the cylindrical transducer. This is indicated mathematically as follows:

$$
f^{(C)}(\theta, z)=f(r, \theta, z) \delta\left(r-r_{0}\right)
$$

where $\delta$ is the Dirac-Delta function and $f^{(C)}(\theta, z)$ is a transverse object function. Thus (4.8) can be simplified as:

$$
\tilde{R}^{\prime}\left(r_{0}, k_{\theta}, k_{z}\right)=\int_{-\infty}^{\infty} \tilde{R}\left(k, k_{\theta}, k_{z}\right) \tilde{H}^{*}\left(k, r_{0}, k_{\theta}, k_{z}\right) d k
$$

Following the discussion in 3D case above, we obtain the constructed image:

$$
f^{\prime}\left(r_{0}, \theta, z\right)=F_{k_{\theta}, k_{z}}^{-1}\left\{\tilde{R}^{\prime}\left(r_{0}, k_{\theta}, k_{z}\right)\right\} \approx f\left(r_{0}, \theta, z\right)
$$

To summarize, the steps to construct a C-mode image are as follows: Perform a 2D Fourier transform of received echo signals to get the spectrum in terms of $\theta$ and $z$, multiply the results with $\tilde{H}^{*}\left(k, r_{0}, k_{\theta}, k_{z}\right)$ and integrate over $k$, and then the images is constructed with an inverse Fourier transform over both $k_{\theta}$ and $k_{z}$.

A similar approach can be used to construct a 2D B-mode image, i.e., an image along both $r$ and $\theta$ dimension with a fixed $z$ (or object is uniform along $z$ ). Under this condition, 
$f(r, \theta, z)$ can be replaced with $f(r, \theta)$. For simplicity, without loosing generality, we assume $z=0$. From (4.4), we have:

$$
R\left(k, \theta_{e}\right)=\int_{r_{e}}^{\infty} f(r, \theta) \otimes_{\theta} h(k, r, \theta) d r
$$

After the Fourier transform of $R\left(k, \theta_{e}\right)$ in terms of $\theta_{e}$, from (8), we obtain:

$$
\tilde{R}^{\prime}\left(r^{\prime}, k_{\theta}\right)=\int_{-\infty}^{\infty} \tilde{R}\left(k, k_{\theta}\right) \tilde{H}^{*}\left(k, r^{\prime}, k_{\theta}\right) d k
$$

Instead of 2D, 1D inverse Fourier transform is used to construct the image:

$$
f^{\prime}(r, \theta)=F_{k_{\theta}}^{-1}\left\{\tilde{R}^{\prime}\left(r, k_{\theta}\right)\right\} \approx f(r, \theta)
$$

\subsection{Simulation results}

The simulation of pulse-echo imaging is performed in the three-dimension. In the simulations, Rayleigh-Sommerfeld diffraction formula is used. The parameters of the cylindrical transducer for the simulation are as follows (Fig.4.1): The transducer is broadband and its center frequency is $1.5 \mathrm{MHz}$. The bandwidth of the transducer is about $81 \%$ of the center frequency. [assume that the combined transmit and receive transfer function is proportional to the Blackman window function], The background medium is assumed to be water that has a speed of sound of $1500 \mathrm{~m} / \mathrm{s}$ given the wavelength of $1 \mathrm{~mm}$ at the central frequency. The objects are assumed to be composed of point scatters. The radius of the cylindrical transducer is $40 \mathrm{~mm}$; the range of the angle is from $-45^{0}$ to $45^{\circ}$, and the range of transducer along the $z$-axis is from $-25 \mathrm{~mm}$ to $25 \mathrm{~mm}$. The inter-element distance of the array transducer is assumed to be $0.7087^{\circ}$ along angle direction and $0.3927 \mathrm{~mm}$ along $z$ direction. So the element number of the discrete transducer is $128 \times 128$. In transmission, all the array elements are connected electronically to transmit the cylindrical wave, which is approximated by zero order Hankle function. Echoes from object (Fig.4.1) are received with the same array and processed to construct imaging by the several steps below based on the previous analysis.

1. Do Fourier transform of received signal (see (4.6)) in terms of $\theta$ and $z$, i.e., $\tilde{R}\left(k, k_{\theta}, k_{z}\right)=F_{\theta, z}\{R(k, \theta, z)\}$

2. Multiply the results with the known function, $\tilde{H}^{*}\left(k, r^{\prime}, k_{\theta}, k_{z}\right)$ (see (4.7)).

3. Integrate the result over $k$ according to (4.8).

4. Performing an inverse Fourier transform according to (4.10) to construct image.

The objects used for the construction are shown in Fig.4.1 and Fig.4.4 and are composed of either a single point scatter or nine point scatters which form a cross shape in the plane defined by $r-\theta$ and the plane defined by $z-\theta$. The geometry center of the object is located at $(r, \theta, z)=\left(r_{0}, 0,0\right)$. Results of the pulse-echo images are given in Fig.4.2, Fig.4.3 and Fig.4.5,

Fig.4.6

Fig.4.2d, e and f show the image for one scatter which position is $(r, \theta, z)=(90 \mathrm{~mm}, 0,0)$. Because the radius of the transducer is $40 \mathrm{~mm}$, the nearest distance between the scatter and 
the surface of transducer is $50 \mathrm{~mm}$. To see the sidelobes of the constructed images, line plots of the single point scatter along $r, z$ and $\theta$ direction in the $z-\theta$ plane, $r-\theta$ plane and $z-r$ plane are shown in Fig.4.3. From the results it can be seen that imaging is very similar to the result of PSF. This means that the approximations (4.10) due to a finite temporal bandwidth and limited spatial Fourier-domain coverage that are typical in medical ultrasonic imaging do not significantly affect the equality of constructed images in terms of spatial resolutions, sidelobes, and contrast.

Fig.4.5 shows the images for nine scatters. The nearest distance between surface of the transducer and the geometry center of the object is chosen to be $50 \mathrm{~mm}\left(r_{0}=90 \mathrm{~mm}\right), 100 \mathrm{~mm}$ $\left(r_{0}=140 \mathrm{~mm}\right)$ and $200 \mathrm{~mm}\left(r_{0}=240 \mathrm{~mm}\right)$, respectively. An interesting phenomenon is that simulation shows the sidelobes and resolution of the images of the object, of which the geometry center is at different distance, is nearly the same (Fig.4.6). The reason is that the transmitted field keeps the same form as the theory prediction along $r$ direction if the filed is cylindrical wave. Though the side lobe and resolution in the $r$ and $\theta$ direction is the same for the larger area, the sidelobe rises and the resolution is lower in the regular coordinate system when $r$ becomes larger because of the relationships $z=r \cos (\theta), x=r \sin (\theta)$.

\subsection{Conclusion}

In this section a new 3D images system in cylindrical coordinate has been developed with cylindrical wave beams (zero order Hankle function). This computation is much less than conventional delay and sum method, so the method has a potential to achieve a high image frame rate and can be implemented with relatively simple inexpensive hardware because the FFT and IFFT algorithm can be used. Computer simulation with the new method has been carried out to construct 3D images. Though the aperture geometry of the transducer is only part of a cylinder, and the transmitted filed is not exact zero order Hankle function, the results of the simulation still match theoretical prediction. So the new imaging method is robust and is not sensitive to various limitations imposed by practical system. In addition, though the discussion above is mainly for 2D cylindrical transducer in three-dimension in the cylindrical coordinate system, the method can be used directly for the $1 \mathrm{D}$ curve transducer in two-dimension in the polar coordinate system obviously.

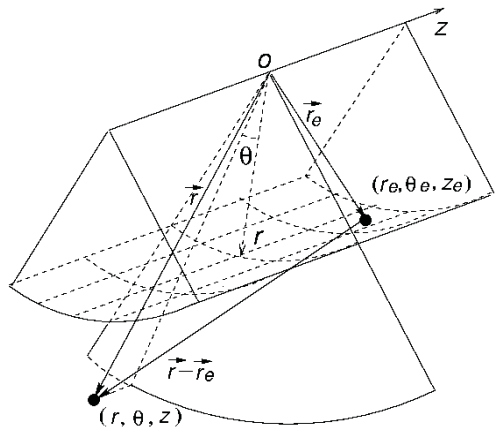

Fig. 4.1. Transducer in the cylindrical coordinate system. The radius of the transducer is $r_{e}=40 \mathrm{~mm}$, and the range of axial axis $z_{e}$ is from $-25 \mathrm{~mm} \sim 25 \mathrm{~mm}$, the range of azimuthal angle $\theta_{e}$ is from $-45^{0}$ to $45^{0}$. There are $N_{z} N_{\theta}=128 \times 128$ elements 


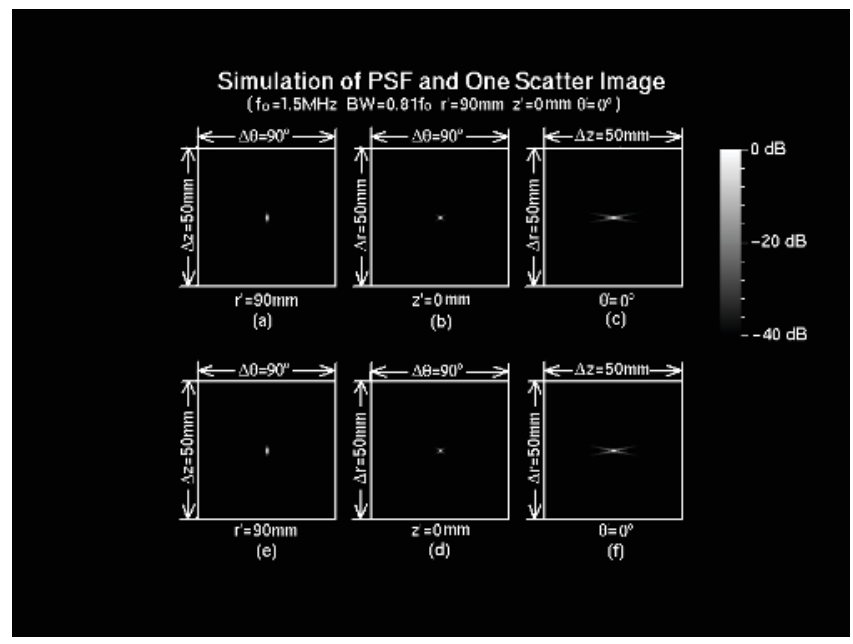

Fig. 4.2. Calculated PSF, $g^{\prime}\left(r-r^{\prime}, \theta, z\right)=F_{k_{\theta}, k_{z}}^{-1}\left\{G^{\prime}\left(r-r^{\prime}, k_{\theta}, k_{z}\right)\right\}$, and the image constructed from one scatterer (Fig. 4.1) where $r^{\prime}=90 \mathrm{~mm}$. (a) shows the distribution of PSF in the plane $(\theta, z)_{r=90 \mathrm{~mm}}$.(b) shows the distribution of PSF in the plane $(r, \theta)_{z=0}$. (c) shows the distribution of PSF in the plane $(r, z)_{\theta=0}$. (d) shows the constructed image of the scatter in the plane $(\theta, z)_{r=90}$. (e) shows the image of the scatter in the plane $(r, \theta)_{z=0}$. (f) shows the image of the scatter in the plane $(r, z)_{\theta=0}$. The images are log compressed over $40 \mathrm{db}$

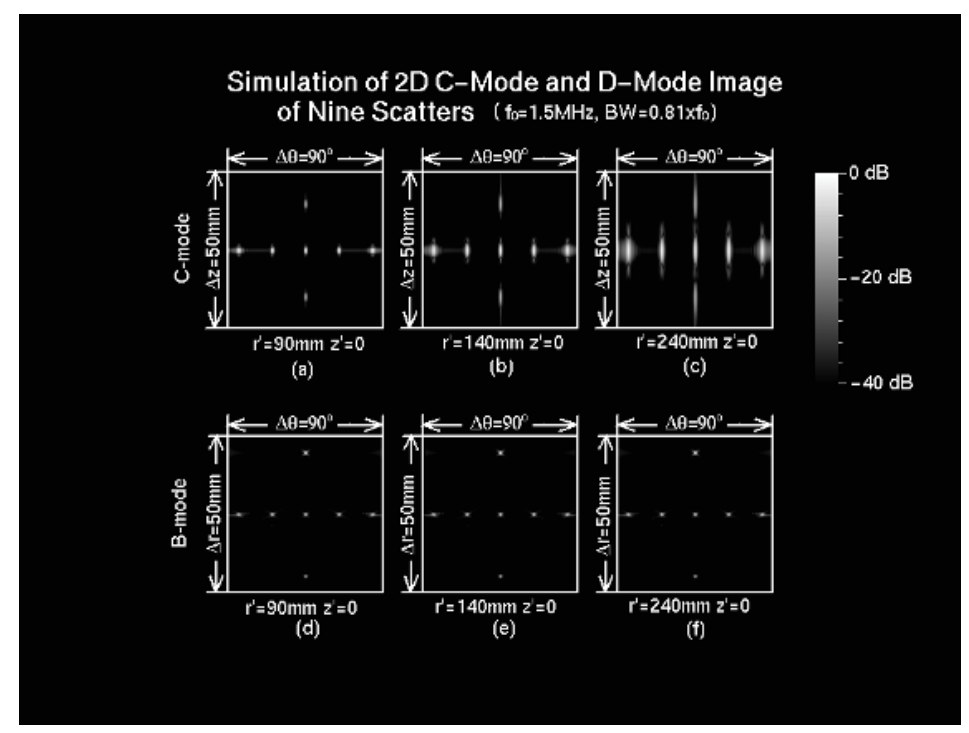

Fig. 4.5. The images for Fig.4.4. (a), (b) and (c) show images where the geometry center is equal to $90 \mathrm{~mm}, 140 \mathrm{~mm}$ and $240 \mathrm{~mm}$ by C-mode $(z-\theta$ plane) respectively. (d),(e) and (f) show images where the geometry center is equal to $90 \mathrm{~mm}, 140 \mathrm{~mm}$ and $240 \mathrm{~mm}$ by Bmode( $r-\theta$ plane), respectively. The images are log compressed over $40 \mathrm{db}$ 
Sidelobe Plots of PSF and One Scatter

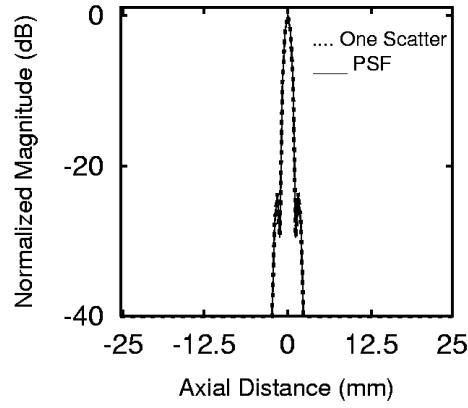

(a)

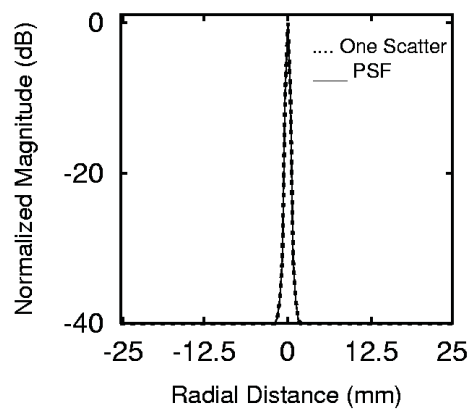

(c)

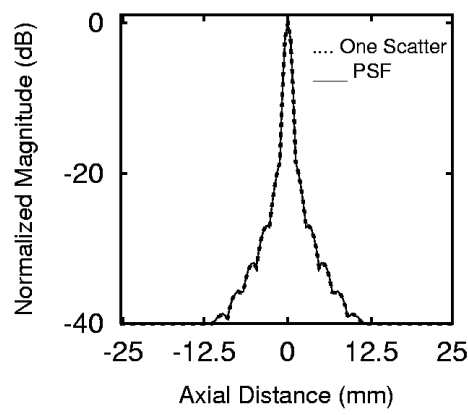

(e)

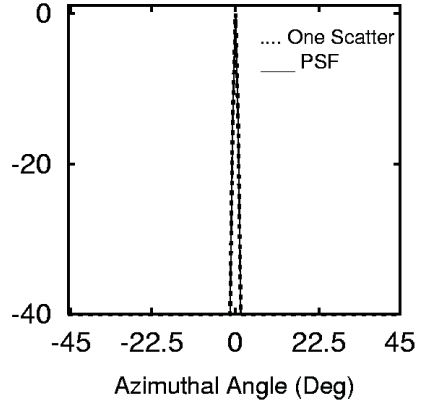

(b)

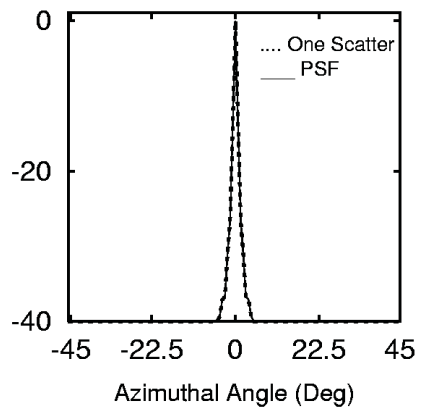

(d)

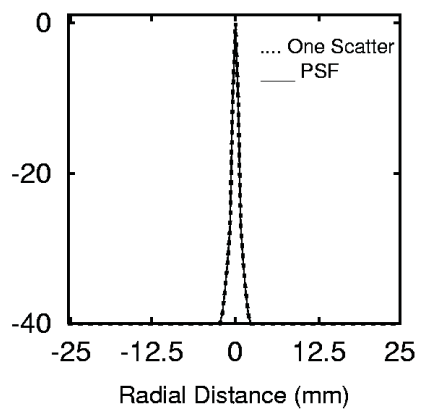

(f)

Fig. 4.3. Sidelobe of PSF and constructed image of one scatter in Fig.4.2. (a) and (b) show sidelobe in the plane $(\theta, z)_{r=90}$ along $z$ direction and $\theta$ direction, respectively. (c) and (d) show sidelobe in the plane $(r, \theta)_{z=0}$ along $r$ direction and $\theta$ direction, respectively. (e) and (f) show sidelobe in the plane $(r, \theta)_{z=0}$ along $z$ direction and $r$ direction, respectively 


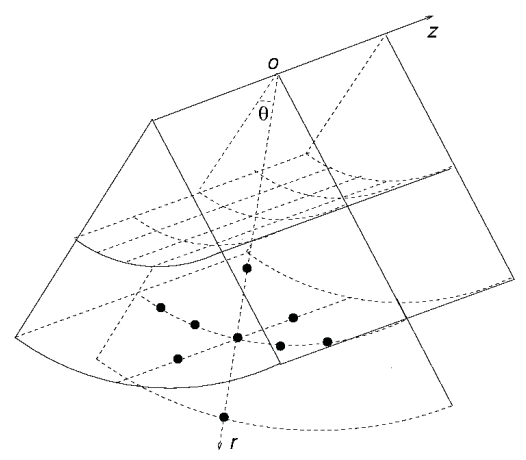

Fig. 4.4. The objects used for construction of images, which contains nine scatters and the geometry center is $(r, \theta, z)=(90,0,0),(140,0,0)$ or $(240,0,0)$

Sidelobe Plot of Nine Scatters

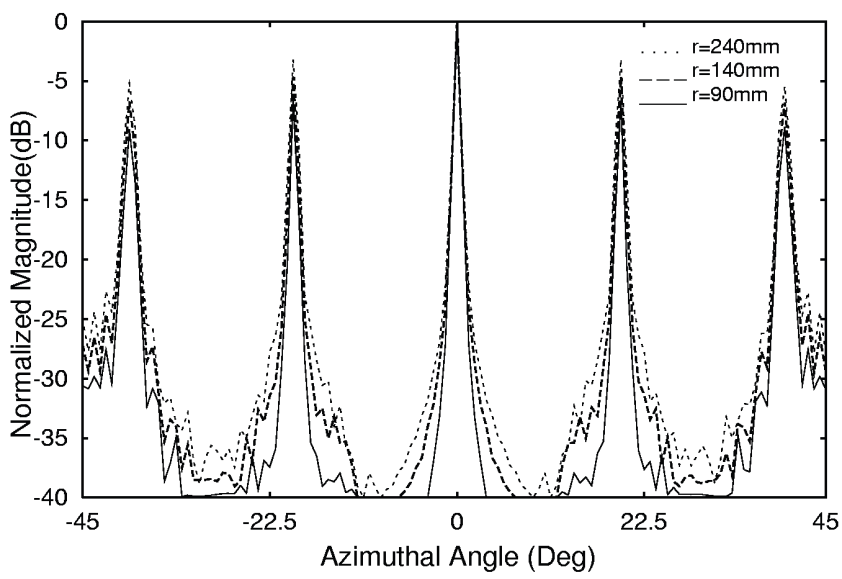

Fig. 4.6. Plots line shows sidelobe for Fig.4.5 along $\theta$ direction in the B-mode image

\section{References}

Jian-yu Lu. (1997). 2D and 3D high frame rate imaging with limited diffraction beams, IEEE Transactions on Ultrasonics, Ferroelectrics, and Frequency Control, Vol.44, No.4, (1997), pp. 839-856, ISSN 0885-3010

$\mathrm{Hu}$, Peng; Angning Yu. (2008). High Frame Rate Ultrasonic Imaging through Fourier Transform Using an Arbitrary Known Transmission, Elsevier-Computers and Electrical Engineering, Vol.34( 2008), pp. 141-147, ISSN 0045-7906

$\mathrm{Hu}$, Peng (2007). A new model of ultrasonic imaging system based on plane wave transmission and angular spectrum propagation principle, 2007 IEEE International Conference on Integration Technology, pp. 307-310, ISBN 1-4244-1092-4, Shenzhen, China, March 20-24, 2007 
Hu, Peng; Jianyu Lu \& Xue Mei Han. (2006). High Frame Rate Ultrasonic Imaging System Based on the Angular Spectrum Principle, Elsevier-Ultrasonics, Vol.44 (2006), pp. 97-99, ISSN 0041-624X

$\mathrm{Hu}$, Peng; Jianyu Lu (2002). High Frame Rate 2D and 3D Imaging with a Curved or Cylindrical Array, 2002 IEEE Ultrasonics Symposium, pp. 1725-1728, ISBN 0-78037582-3, Munich, Germany, October 8-11, 2002 


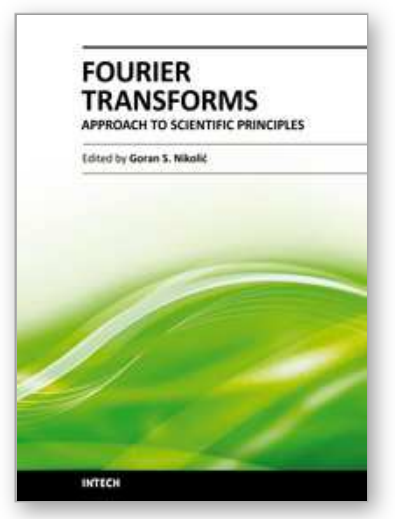

\author{
Fourier Transforms - Approach to Scientific Principles \\ Edited by Prof. Goran Nikolic
}

ISBN 978-953-307-231-9

Hard cover, 468 pages

Publisher InTech

Published online 11, April, 2011

Published in print edition April, 2011

This book aims to provide information about Fourier transform to those needing to use infrared spectroscopy, by explaining the fundamental aspects of the Fourier transform, and techniques for analyzing infrared data obtained for a wide number of materials. It summarizes the theory, instrumentation, methodology, techniques and application of FTIR spectroscopy, and improves the performance and quality of FTIR spectrophotometers.

\title{
How to reference
}

In order to correctly reference this scholarly work, feel free to copy and paste the following:

Hu Peng (2011). High Frame Rate Ultrasonic Imaging through Fourier Transform using an Arbitrary Known Transmission Field, Fourier Transforms - Approach to Scientific Principles, Prof. Goran Nikolic (Ed.), ISBN: 978-953-307-231-9, InTech, Available from: http://www.intechopen.com/books/fourier-transforms-approach-toscientific-principles/high-frame-rate-ultrasonic-imaging-through-fourier-transform-using-an-arbitrary-knowntransmission- $f$

\section{INTECH}

open science | open minds

\author{
InTech Europe \\ University Campus STeP Ri \\ Slavka Krautzeka 83/A \\ 51000 Rijeka, Croatia \\ Phone: +385 (51) 770447 \\ Fax: +385 (51) 686166 \\ www.intechopen.com
}

\author{
InTech China \\ Unit 405, Office Block, Hotel Equatorial Shanghai \\ No.65, Yan An Road (West), Shanghai, 200040, China \\ 中国上海市延安西路65号上海国际贵都大饭店办公楼 405 单元 \\ Phone: +86-21-62489820 \\ Fax: $+86-21-62489821$
}


(C) 2011 The Author(s). Licensee IntechOpen. This chapter is distributed under the terms of the Creative Commons Attribution-NonCommercialShareAlike-3.0 License, which permits use, distribution and reproduction for non-commercial purposes, provided the original is properly cited and derivative works building on this content are distributed under the same license. 